\title{
RECTANGULAR WELL AS PERTURBATION
}

\author{
Mariusz Dudek , Stefan Giller弝 \\ and \\ Piotr Milczarski抿 \\ Theoretical Physics Department II, University of Łódź, \\ Pomorska 149/153, 90-236 Łódź, Poland \\ e-mail: † sgiller@krysia.uni.lodz.pl \\ $\ddagger$ jezykmil@krysia.uni.lodz.pl
}

\begin{abstract}
We discuss a finite rectangular well as a perturbation for the infinite one with a depth $\lambda^{2}$ of the former as a perturbation parameter. In particular we consider a behaviour of energy levels in the well as functions of complex $\lambda$. It is found that all the levels of the same parity are defined on infinitely sheeted Riemann surfaces which topological structures are described in details. These structures differ considerably from those found in models investigated earlier [1, 4, 8, 9, 10]. It is shown that perturbation series for all the levels converge what is in a contrast with the known results of Bender and $\mathrm{Wu}$ [1]. The last property is shown to hold also for the infinite rectangular well with Dirac delta barrier as a perturbation considered earlier by Ushveridze [ 1 .
\end{abstract}

PACS number(s): 03.65.-W

Key Words: perturbation theory, convergent perturbation series, energy level crossing

\footnotetext{
${ }^{1}$ Supported by KBN 2PO3B 07610

${ }^{2}$ Supported by the Łódź University Grant No 580
} 


\section{Introduction}

Since the papers of Bender and $\mathrm{Wu}$ [1] we have known why the perturbation series were in general divergent. We have known also that in many cases investigations of perturbation series could be reduced to the investigations of the corresponding semiclassical series [2]. It was also realized that the divergent perturbation series could be summed and one of the summation methods applied here was very often the Borel one [2, 3].

One of byproducts of these investigations was a discovery of so called level crossing i.e. of the fact that in the case of confining polynomial potentials all the discrete energy levels they produce or only groups of them are no longer isolated of each other if considered as functions of a perturbation parameter [1, 3, 8, 9] i.e. the levels inside each group appear as branches of a ramified functions of the perturbation parameter considered as a complex variable. Being more precise the latter statement means that each energy level belonging to a group considered as a function of a real perturbation parameter can be analytically continued into the complex plane of the parameter so that any energy level of the group can be reached by the analytic continuation procedure of some arbitrary chosen level belonging to the group. This means also that the complex plane of the perturbation parameter converts rather into some (more or less) complicated Riemann surface.

It is also well known [7] that it is an existing symmetry group of the Hamiltonian considered which is completely responsible for a decay of the energy spectrum into disjoint (with respect to analytic continuation) groups of them. Therefore, a degree of complication of respective Riemann surfaces on which the energy levels are defined can give us an information about an existence of the relevant symmetry group i.e. the more levels appear as branches defined on respective sheets of the same Riemann surface the less rich a relevant symmetry group has to be. In particular if the corresponding Riemann surfaces are all finitely sheeted (i.e. if there are finite numbers of energy levels attached to each of them) then they have to be defined by some algebraic conditions relating energies and a perturbation parameter, with the conditions being a clear sign of the existence of an underlying symmetry group [7].

There are only a few examples of the analysis described above in the case when the relevant Riemann surface is infinitely sheeted [1, 4]. The analysis performed is more or less numerical. This is mostly because it is very difficult to find an example of potential providing us with a closed functional form of the quantization condition being simultaneously sufficiently simple to perform an analysis in a 'classical', non numerical way. According to these investigations a Riemann surface topology corresponding to a given perturbation parameter i.e. loci of its branch points seems to be still mysterious and depending on a parameter chosen so needing still further studies.

In this paper we will consider a possibly simple but non trivial example of an Hamiltonian provided by the familiar rectangular well of a finite height which allows us for such a classical analysis. As a perturbation parameter in this example we will choose its height $V=\lambda^{2}>$ 0 ; strictly speaking the square root of it). The perturbed potential is then the infinite rectangular well approached when $V^{-1} \rightarrow 0$. There are several basic properties which differ the case of the finite rectangular well from the ones considered earlier. First it is just a finite number of energy levels existing for a given $\lambda$ but varying with $\lambda$ so that a potentially infinite number of levels can appear when $\lambda^{-1} \rightarrow 0$. The remaining properties are enumerated as points 2., 4. and 5 . below.

The rectangular well is not an analytical potential and as such it provides us also with a non analytical quantization condition. However an analytical extension of the latter into 
complex values of the quantities considered is possible and results of the relevant analysis are the following:

1. The system of energy levels of the well decays into two disjoint families (of different parities) with the levels inside each of the group being analytical continuations of each other with respect to the perturbation parameter;

2. The perturbation series for each level is convergent to the level itself i.e. the property which is quite opposite to that of Bender and $\mathrm{Wu}$ for the unharmonic oscillator case. As such they are trivially Borel summable;

3. The Riemann surfaces for both the groups of levels are infinitely sheeted and their branch point structures can be understood by some simple properties of both the quantization conditions.

4. The energy level poles existing in the complex momentum plane corresponding to the case are accompanied by poles which do not represent nor discrete nor resonant parts of the energy spectrum (in particular because resonances are absent in the case of the finite rectangular well independently of whether the latter is a real well (for real $\lambda$ ) or is a rectangular barrier (for imaginary $\lambda$ )). These second sort of poles we shall call pseudoenergy levels.

5. The level crossing which happens for real $\lambda$ is not between two real energies but just between an energy and its pseudoenergy partner.

We will reconsider also an example of a numerical analysis performed earlier by Ushveridze [4] to show that its 'classical' analysis is possible in its full size confirming the main results of the author mentioned but completed them with such an important conclusion as a convergence of both perturbation series corresponding to the weak and the strong couplings to the Dirac delta perturbation used in the example.

Another goal of our investigations was looking for rules governing distributions of energy level branch points on the perturbation parameter Riemann surface. It has been demonstrated by Ushveridze 细 that one can predict an existence of energy level crossing as well as arrange the crossing to join more than two levels but as in the case of the anharmonic potential [1] distributions of the corresponding branch points seemed to be unpredictable a priori to direct calculations.

Our main conclusion in this respect is similar. Indeed such distributions of branch points is strongly model (potential) dependent and it seems that only some crude rules can be formulated to predict their presence or absence in some domains of the underlying Riemann surface.

\section{Finite rectangular well as a perturbation}

\subsection{Analytic properties of energy levels as functions of perturbation parameter}

A finite rectangular well as a perturbation sounds a little bit exotically but it can be considered as such for the infinite one in the same way as for example a finite potential well:

$$
\frac{1}{2} \frac{\omega^{2}}{\alpha^{2}}\left(1-\frac{1}{\cosh (\alpha x)}\right)
$$

being a perturbation for the harmonic one i.e. we get the latter from (2.1) for $\alpha \rightarrow 0$. 
In the case of a finite rectangular potential given by:

$$
V(x)= \begin{cases}V(>0) & \text { for }|x|>1 \\ 0 & \text { for }|x|<1\end{cases}
$$

a perturbation parameter can be chosen to be $\lambda^{2}=V$ which together with the rescaled energy $E / V \equiv z^{2}$ leads us to the following quantization conditions $[5]$ :

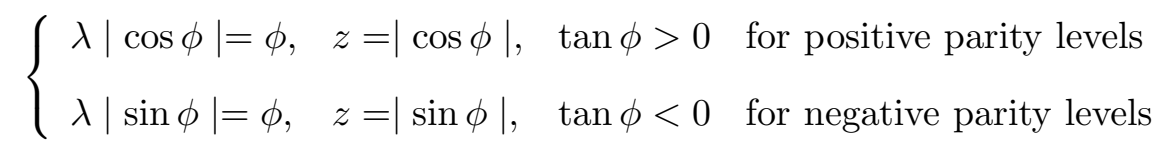

All (positive) solutions $\phi_{k}(\lambda), k=0,1,2, \ldots$, to Eq.'s (2.3) are represented picturesquely in Fig.1 as given by the points of intersections of the straight line $\phi / \lambda$ with the right arcs of $|\sin (\phi)|$ and $|\cos (\phi)|$ functions. The corresponding solutions for energy levels are then get as $E_{k}(\lambda)=\lambda^{2} z^{2}\left(\phi_{k}(\lambda)\right)$. It is just a dependence of $E_{k}$ 's on $\lambda$ considered as a complex parameter which is the main interest of this paper.

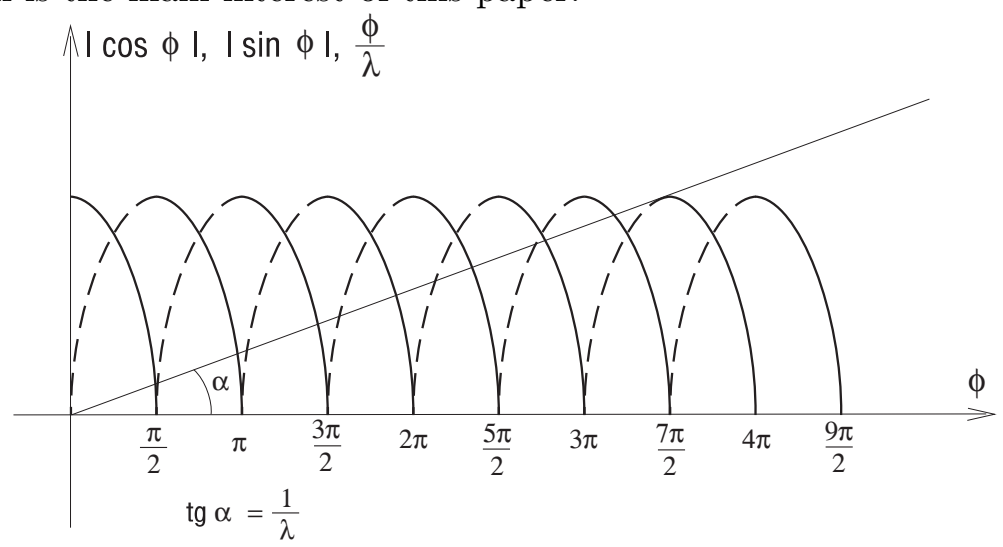

Fig.1 The $\phi$ angles chosen by the quantization conditions (2.3)

To investigate this dependence it is however necessary to make an analytical continuation of the conditions (2.3) into the complex $\lambda$. Such a continuation of Eq.'s (2.3) is possible when dropping the absolute value marks in (2.3) what provides us with following analytic conditions:

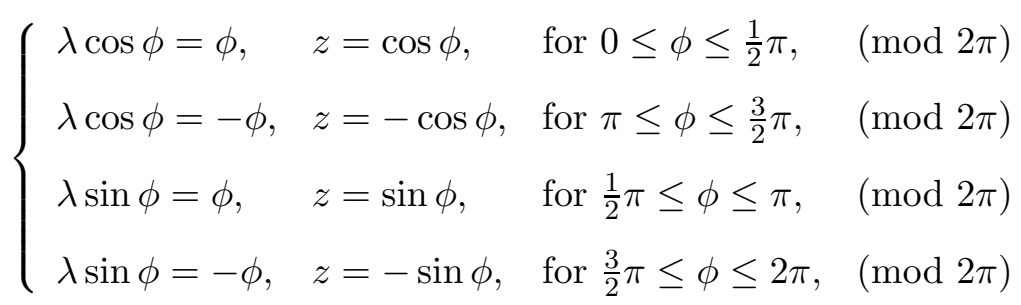

equivalent to (2.3) for positive $\phi$.

According to (2.4) the energy spectrum for the potential (2.2) is formally divided into four groups. Each group contains every forth members of the spectrum starting from $E_{1}^{+}$, $E_{3}^{+}, E_{2}^{-}, E_{4}^{-}$energy levels in the corresponding group. The energy levels in the groups are defined by the conditions (2.4) in the order mentioned. 
Dropping further the restrictions for the ranges of changing $\phi$ in the conditions (2.4) we get fully analytical quantization conditions but describing rather different spectra with respect to which our original ones are only parts of them. This is however the necessary price for investigating the complex analytical dependence of energy levels on $\lambda$.

When doing it however we observe that for both the parity levels it is enough to continue analytically only the firsts of the corresponding conditions (2.4) depriving them of the corresponding restrictions for $\phi$ (i.e. $\phi$ can now take any complex value in these conditions). This is because corresponding solutions $\phi^{ \pm}(\lambda)$ to the first conditions generate solutions $\phi^{ \pm}(-\lambda)$ to the second ones. (In fact for the even parity both the solutions almost coincide since $\left.\phi^{+}(-\lambda)=-\phi^{+}(\lambda)\right)$. Therefore we see that all energy levels of both parities: $E_{k}^{+}(\lambda)=\lambda^{2} \cos ^{2} \phi_{k}^{+}(\lambda)$ and $E_{k}^{-}(\lambda)=\lambda^{2} \sin ^{2} \phi_{k}^{-}(\lambda)$ can be obtained by solving only the first conditions (2.4) of the corresponding parities and performing analytic continuations in $\lambda$ from its positive to its negative values.

We will analyze first the even parity group determining the ground state energy level. An analysis of the odd parity case is quite similar.

\section{a) Even parity energy spectrum case}

Making in the first of the conditions (2.4) a change of variable $e^{i \phi}=\sigma$ we get instead:

$$
\left\{\begin{array}{l}
\lambda^{+}=-\frac{2 i \sigma \ln \sigma}{\sigma^{2}+1} \\
z^{+}=\frac{1}{2}\left(\sigma+\frac{1}{\sigma}\right)
\end{array}\right.
$$

Since a dependence of $z^{+}$on $\sigma$ as given by (2.4) is rather simple then to get the corresponding dependence of $z^{+}$on $\lambda$ it is necessary to invert the dependence of the latter variable on $\sigma$ as shown in (2.5). To do this one needs to know:

$1^{0}$ The Riemann surface structure for $\lambda^{+}(\sigma)$;

$2^{0}$ The loci of all zeros of $\lambda^{+^{\prime}}(\sigma)$ on the surface; and

$3^{0}$ A pattern of lines $\operatorname{Re} \lambda^{+}=$const and $\operatorname{Im} \lambda^{+}=$const on the surface.

As it follows from (2.5) the Riemann surface structure for $\lambda^{+}(\sigma)$ is determined by:

a. The logarithmic branch point at $\sigma=0$; and

b. A pair of simple poles at $\sigma= \pm i$ located on every sheet the latter being generated in an infinite number by the logarithm.

Zeros of $\lambda^{+^{\prime}}(\sigma)$ are determined by the following equation:

$$
\ln \sigma=\frac{\sigma+1}{\sigma-1}
$$

Putting $e^{i \phi+y}=\sigma$ ( $\sigma$ is now an arbitrary complex number on the surface) and assuming that $\phi, y \neq 0$ we transform (2.6) into:

$$
\left\{\begin{array}{l}
\frac{\sin 2 \phi}{2 \phi}=-\frac{\sinh 2 y}{2 y} \\
\cos 2 \phi=-\frac{\sinh 2 y}{2 y}+\cosh 2 y \\
\phi, y \neq 0
\end{array}\right.
$$


from which it follows that all zeros of $\lambda^{+^{\prime}}(\sigma)$ have to lie on the circle $|\sigma|=1$ and/or on the real half axis $\phi=0$. Therefore the corresponding conditions for them are:

$$
\left\{\begin{array}{l}
\cot \phi=-\phi \\
\ln \sigma=\frac{\sigma+1}{\sigma-1}
\end{array}\right.
$$

Solutions to the first of Eq.'s (2.8) (in fact infinitely many of them) are given therefore as the intersection points of the functions $\cot \phi$ and $-\phi$. The form of the condition can be easily identified as the one for the straight line $\phi / \lambda$ of Fig. 1 to be tangent to $\cos \phi$. Therefore the points have to lie close to $\phi=2 k \pi$ ones, $k=1,2, \ldots$, on the left to them, and close to $\phi=k \pi$, $k=-1,-3, \ldots$, on the right to the latter.

Two real solutions to the second of Eq.'s (2.8) are placed on both the sides of $\sigma=1$ at $\sigma_{1} \approx 3.32$ and at $\sigma_{1}^{-1} \approx 0.301$.

Let us note that the points on the unit circle at $\phi=k \pi, k=-1,+2,-3,+4, \ldots$, and the point $\sigma=1$ are all the physical 'thresholds' for successive appearing of the corresponding even parity energy levels according to changing $\lambda$ from zero to infinity. There is a temptation to understand the zeros provided by the conditions (2.8) as a shifting of these thresholds from their real physical positions mentioned to their actual ones because of the approximations which the analytical conditions (2.8) effectively are to our rectangular well quantization problem. This shifting of thresholds remains in a deep relation to analytical properties of reflection and transmission coefficients of the corresponding scattering problem arising when energy is higher than $\lambda^{2}$. We will discuss this relation below.

Although the solutions to the condition (2.8) cannot be considered as thresholds for any real energy spectrum case (this is excluded by the absence of the energy level degeneracy in 1-dim SE) we shall consider them as such for convenience and call them pseudothresholds.

Thus the solutions to the first of the conditions (2.8) are the pseudothresholds for the energy levels lying inside the potential above the ground state one. These pseudothresholds as we have mentioned earlier coincide with results of demanding for the line $\phi / \lambda$ in Fig. 1 to be tangent to $\cos \phi$. The latter demand is just the lower limit for $\lambda$ above which the real solutions for the energy levels can exist. Both the solutions at $\sigma_{1}$ and $\sigma_{1}^{-1}$ between which $\lambda^{+}(\sigma)$ is pure imaginary are then the limits for the ground state energy level $E_{1}^{+}$to be a real quantity. However for $E_{1}^{+}(\lambda)$ to be real is not enough to represent a real physical level. The sufficient conditions will be discussed below in section 2.2 .

A pattern of the lines $\operatorname{Re} \lambda^{+}=\operatorname{Im} \lambda^{+}=0$ is sketched on Fig.2 together with the positions of all singular points of $\lambda^{+}(\sigma)$. Since all zeros of $\lambda^{+^{\prime}}(\sigma)$ are simple they result as square root branch point singularities of $\sigma(\lambda)$ on its $\lambda$-Riemann surface. A consequence of this is a perpendicular crossing of two lines $\operatorname{Im} \lambda^{+}=0$ as well as the corresponding two lines $R e \lambda^{+}=0$ in every of such zero. All the lines $\operatorname{Im} \lambda^{+}=0$ emanating outside the unit circle run to infinity on the corresponding sheets of the $\sigma$-Riemann surface parallel asymptotically to the line $R e \sigma=0$. These emanating inside the circle have to cross the logarithmic branch point at $\sigma=0$ being tangent at this point to the line $\operatorname{Re} \sigma=0$. The remaining lines $\operatorname{Im} \lambda^{+}=0$ coincide with all semicircles of the unit circle lying between the poles at $\sigma= \pm i$ on every logarithmic sheet of the $\sigma$-Riemann surface. 


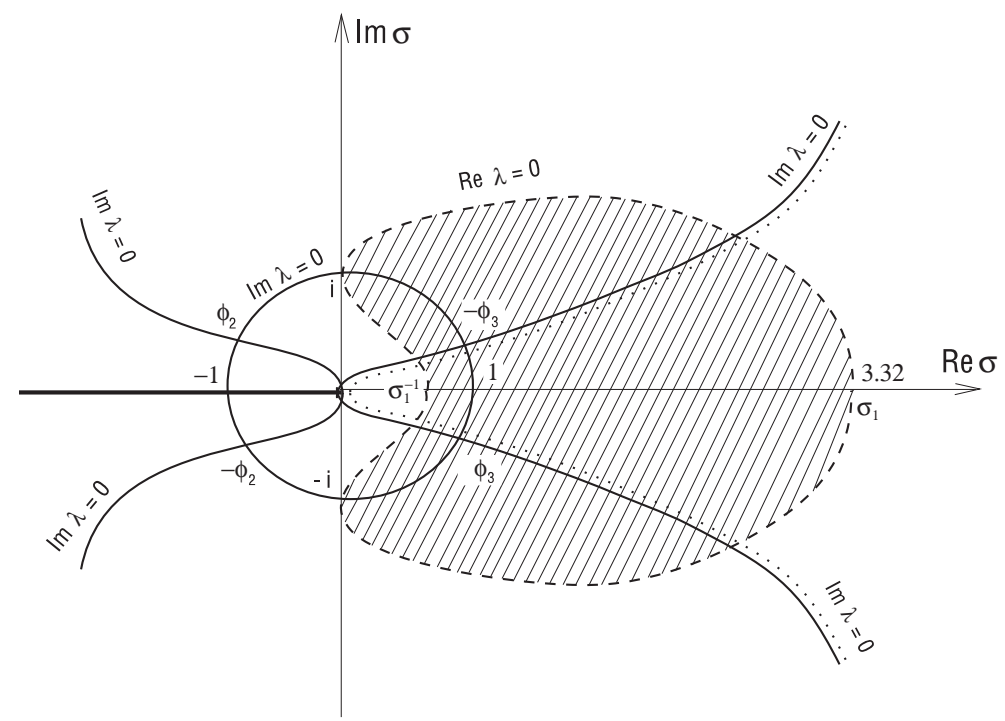

Fig.2 The 'first' sheet of the $\sigma$-Riemann surface corresponding to $\lambda^{+}(\sigma)$

The $\lambda$-Riemann surface on which the inverse function $\sigma^{+}(\lambda)$ is defined is now easy to construct. It is also an infinitely sheeted surface. Its first sheet is a map of the dashed area in Fig. 2 and is shown in Fig. 3a. This is the sheet on which the ground state energy level is defined i.e. for $\lambda$ changing along the real axis of the sheet or along a segment $\left[\lambda_{1},-\lambda_{1}\right] \equiv\left[-\lambda^{+}\left(\sigma_{1}\right), \lambda^{+}\left(\sigma_{1}^{-1}\right)\right]$ on the imaginary axis where the corresponding energy $E_{1}^{+}$is real. On the rest of the sheet the energy is complex.

There are two cuts on the sheet emanating of the two complex conjugate imaginary branch points at $\lambda_{1}=\lambda^{+}\left(\sigma_{1}\right)=-\lambda^{+}\left(\sigma_{1}^{-1}\right)$ and running to infinity along its imaginary axis. The function $\sigma^{+}(\lambda)$ is holomorphic on the sheet approaching $\pm i$ for $\lambda$ escaping to the infinity on the right or on the left half planes of the sheet respectively. The second sheet corresponds to the second energy level. The sheet can be achieved by crossing (in any direction) one of the two cuts described above. Despite these two latter cuts there are another two square root branch points on the sheet lying on the real axis at $\lambda= \pm \lambda_{2}= \pm \lambda^{+}\left(e^{-i \phi_{2}}\right)=\mp \phi_{2} / \cos \phi_{2}$. The energy level $E_{2}^{+}$is given by the values of $\lambda$ changing on the both sides of the real axis from the real branch points mentioned up to the corresponding infinities. On the $\sigma$-Riemann surface these ranges of changing $\lambda$ correspond to varying $\sigma$ along the two left unit semicircles between the poles at $\sigma= \pm i$.

There is still an additional branch point on the discussed sheet at $\lambda=0$ which is also present at all the other sheets except the first one. It is a common picture of the point $\sigma=0$ and all the infinity points of the $\sigma$-Riemann surface of Fig. 2. Therefore this point gives rise to an infinite branching of $\lambda^{+}(\sigma)$ around it.

Starting from the third energy level every of the levels is represented on two different sheets which can communicate with themselves by the branch point at $\lambda=0$. Each of these two sheets belongs to two different groups of them the latter being generated by the two branch points of the second sheet at $\lambda= \pm \lambda_{2}$ (see Fig. 3b). Let us analyze the group generated by the branch point at $\lambda=\lambda_{2}$. The other group is a twin picture of this one obtained by a transformation $\lambda \rightarrow-\lambda$.

Going around $\lambda_{2}$ through the cut $A_{2} / \bar{B}_{2}$ we find ourselves on the sheet shown in Fig. 3c. There is an additional cut on the sheet generated by the branch point at $\lambda=\lambda_{3}=$ 
$\lambda^{+}\left(e^{-i \phi_{3}}\right)=-\phi_{3} / \cos \phi_{3}$. The branch point opens possibility for the fourth and the fifth energy levels to appear. The ranges of the energy levels $E_{3}^{+}$and $E_{4}^{+}$are shown on the figure.
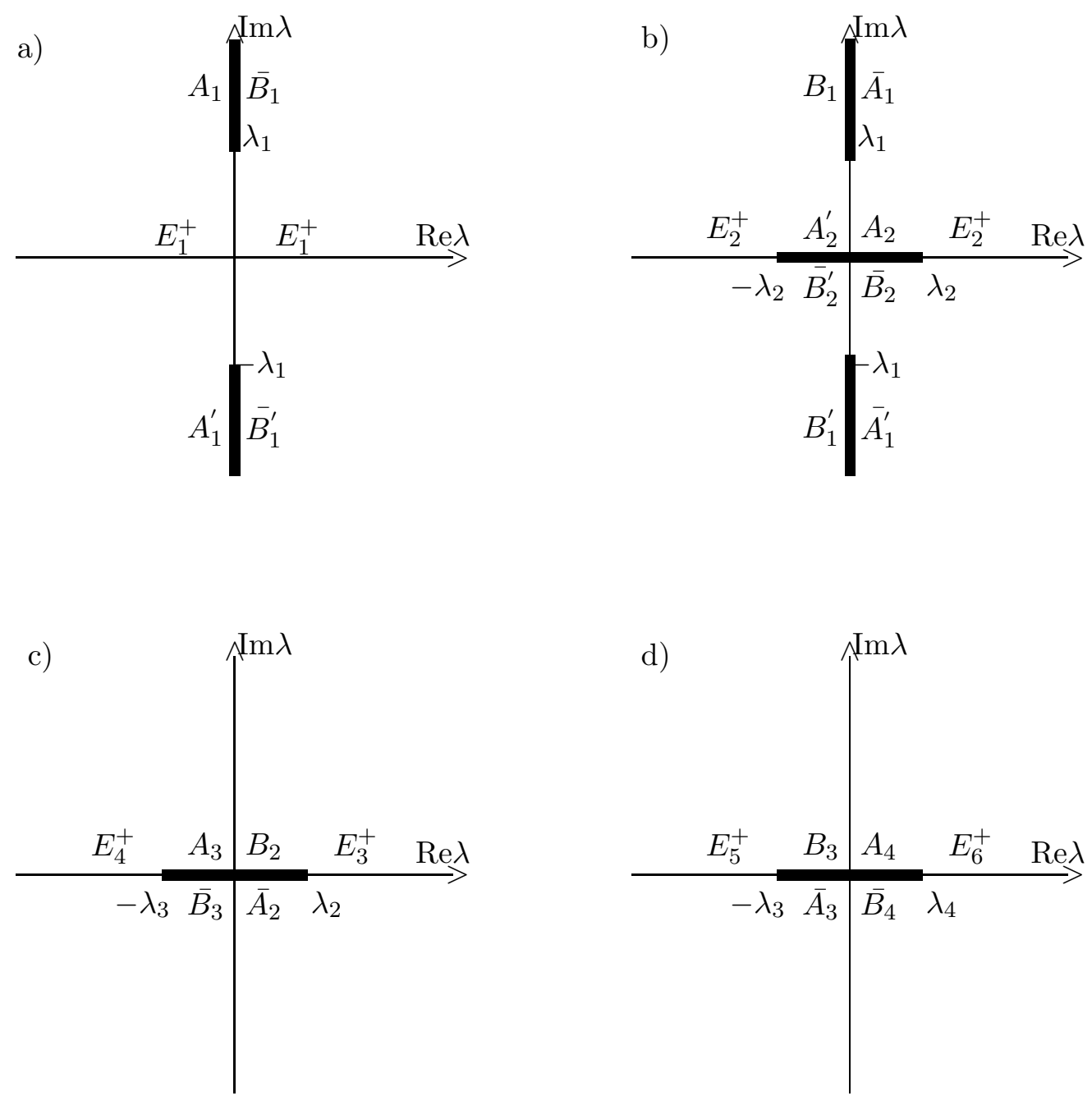

Fig.3 The few first $\lambda$-Riemann surface sheets for $\sigma^{+}(\lambda)\left(E^{+}(\lambda)\right)$

To achieve a sheet corresponding to a pair $E_{5}^{+}, E_{6}^{+}$of the levels we have to cross the cut $A_{3} / \bar{B}_{3}$ in Fig. 3c (in any direction). The sheet is shown in Fig. 3d together with corresponding ranges for the energies.

A full structure of the considered group of sheets of the $\lambda$-Riemann surface is now obvious. The $n^{\text {th }}$ sheet, $\mathrm{n}=3,4, \ldots$, contains three branch points lying at $\lambda=0, \lambda=\lambda_{n-1}=$ $-\phi_{n-1} / \cos \phi_{n-1}$ and at $\lambda=\lambda_{n}=-\phi_{n} / \cos \phi_{n}$. Each of the last two branch points opens a pair of energy levels: $E_{2 n-3}^{+}, E_{2 n-2}^{+}$and $E_{2 n-1}^{+}, E_{2 n}^{+}$, respectively, the levels in each pair 
lying on different sheets. And inversely, the energy levels appear on every sheet in pairs. A $2 n^{\text {th }}$ sheet corresponding to energy levels $E_{4 n-1}^{+}, E_{4 n}^{+}, n=1,2, \ldots$, (see Fig. 4) is cut by two cuts: $B_{2 n} / \bar{A}_{2 n}$ beginning at $\lambda=\lambda_{2 n}=-\phi_{2 n} / \cos \phi_{2 n}>0$ and opening the level $E_{4 n-1}^{+}$and $B_{2 n+1} / \bar{A}_{2 n+1}$ beginning at $\lambda=\lambda_{2 n+1}=-\phi_{2 n+1} / \cos \phi_{2 n+1}<0$ and opening the level $E_{4 n}^{+}$. Both the cuts end at $\lambda=0$. A corresponding sheet for the levels $E_{4 n+1}^{+}, E_{4 n+2}^{+}$is cut from $\lambda_{2 n+1}$ to 0 and from 0 to $\lambda=\lambda_{2 n+2}=-\phi_{2 n+2} / \cos \phi_{2 n+2}>0$ with the latter branch point opening the second of the considered levels (see Fig. 4).

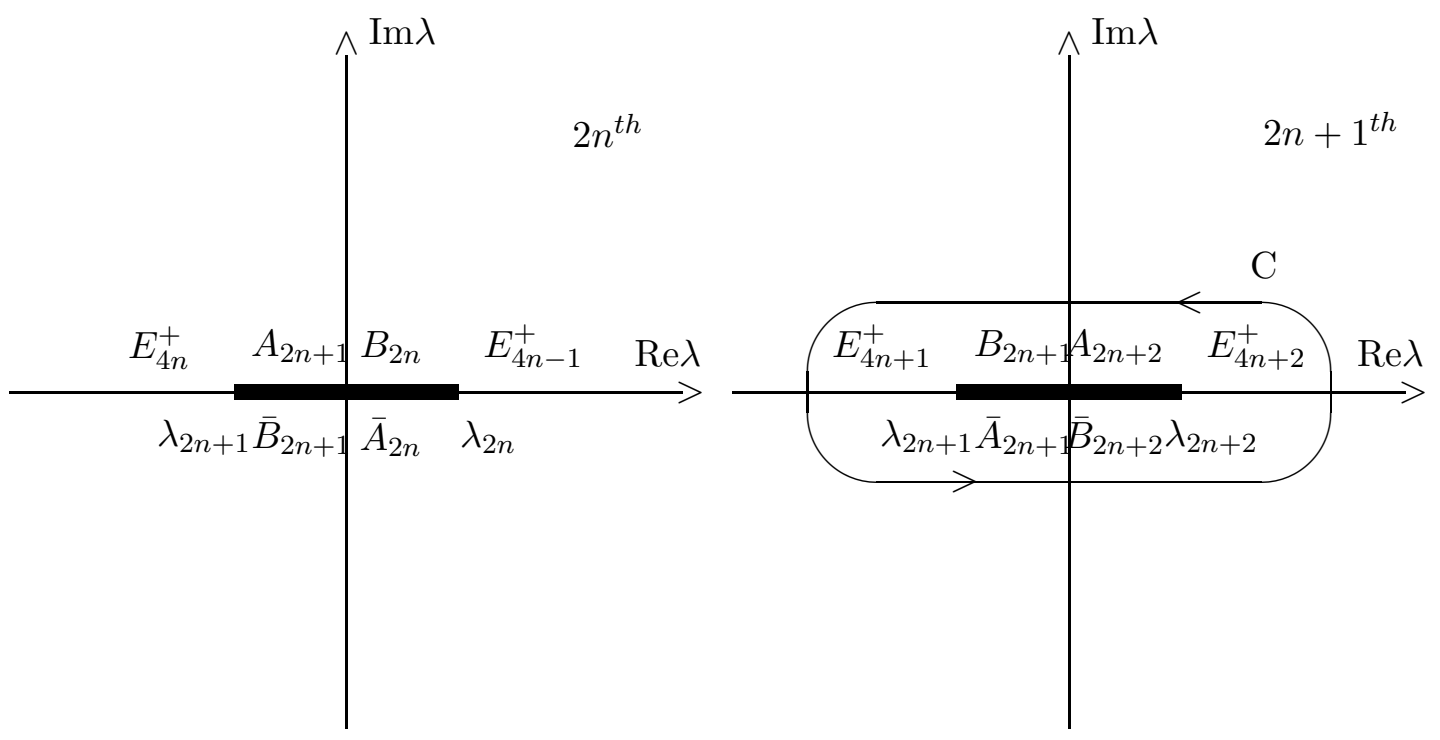

Fig.4 The general structure of $\lambda$-Riemann surface sheets for $\sigma^{+}(\lambda)\left(E^{+}(\lambda)\right)$

The structure of the second group of sheets generated by the branch point at $\lambda=-\lambda_{2}$ is obtained by an inversion: $\lambda \rightarrow-\lambda$ from the first one discussed above.

\section{b) Odd parity energy spectrum case}

Making in the third of the conditions (2.4) a change of variable $\sigma=e^{i \phi}$ we get the condition in the form :

$$
\left\{\begin{array}{l}
\lambda^{-}=\frac{2 \sigma \ln \sigma}{\sigma^{2}-1} \\
z^{-}=\frac{1}{2 i}\left(\sigma-\frac{1}{\sigma}\right)
\end{array}\right.
$$

It follows from (2.9) that $\lambda^{-}(\sigma)$ is a meromorphic function of $\sigma$ on the $\sigma$-Riemann surface with the logarithmic branch point at $\sigma=0$ and with simple poles at $\sigma= \pm 1$ on every logarithmic sheet except the first one where the pole at $\sigma=1$ is absent. The surface is shown in Fig. 5 where the lines $\operatorname{Im} \lambda^{-}(\sigma)=0$ are also shown schematically. Zeros of $\lambda^{{ }^{\prime}}(\sigma)$ are distributed in this case only on the unit circle $|\sigma|=1$ at the point $\sigma=1$ on the first sheet and at points $\sigma=e^{i \phi_{k}}$ with $\phi_{k}\left(=-\phi_{-k}>0\right)$ satisfying the condition $\phi_{ \pm k}=\tan \phi_{ \pm k}$, $k=2, \ldots$, so that the first two of the latter singular points lie on the second sheet of Fig. 5 . 


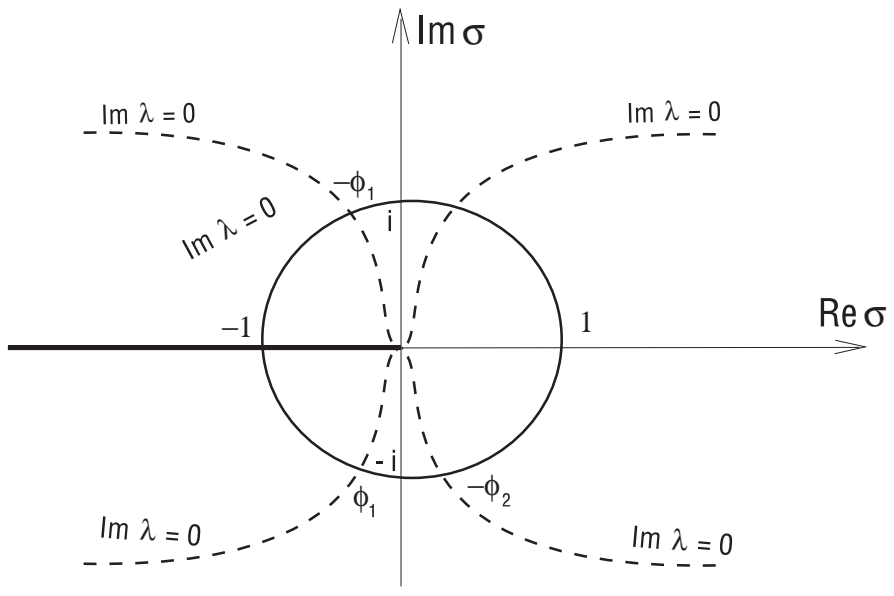

Fig.5 The 'first' sheet of the $\sigma$-Riemann surface corresponding to $\lambda^{-}(\sigma)$

On the other hand the inverse function $\sigma^{-}(\lambda)$ is a holomorphic one on the $\lambda$-Riemann surface with branch points at $\lambda=1$ and at $\lambda_{k}=\lambda^{-}\left(e^{i \phi_{k}}\right)$ which are images of zeros of $\lambda^{-^{\prime}}(\sigma)$.

The spectrum is opened with the level $E_{2}^{-}(\lambda)$ which belongs to the odd parity spectrum of the rectangular well opening it contrary to the next level $E_{3}^{-}(\lambda)$ which does not.

An interesting property of $E_{2}^{-}(\lambda)$ is a singularity it has to have at $\lambda=0$ i.e. the level $E_{2}^{-}(\lambda)$ as a function of $\lambda$ is not bounded from below at this point. This conclusion follows from an observation that although the point $\lambda=1$ is a branch one for $\sigma^{-}(\lambda)$ (below which $\sigma^{-}(\lambda)$ has two values: $\sigma$ and $\sigma^{-1}$ for every $\left.\lambda, 0 \leq \lambda \leq 1\right)$ it is not as such for the level $E_{2}^{-}(\lambda)$. On the other hand $E_{2}^{-}(\lambda)\left(=-\left[\ln \sigma\left(\sigma^{2}+1\right) /\left(\sigma^{2}+1\right)\right]^{2}\right)$ for $0<\lambda<1(0<\sigma<+\propto)$ is real and negative. Therefore a pseudothreshold for the latter appears to be at the point $\lambda=0$ at which $E_{2}^{-}(\lambda)$ becomes infinitely large and negative. Here again we want to stress however that the realness of $E_{2}^{-}(\lambda)$ does not mean automatically its existence as a real physical energy level (see a discussion below, Sec. 2.3) and in the case considered $E_{2}^{-}(\lambda)$ disappears as a physical level below $\lambda=\pi / 2$ as it follows from Fig. 1 .

The remaining levels have already expected properties only.

The level $E_{3}^{-}(\lambda)$ starts with a pseudothreshold $E_{3}^{-}\left(e^{i \phi_{1}}\right)$. A sheet on which both the levels vary is shown in Fig. 6. It emerges as a map of an area of Fig. 5. lying between the real halfline $\sigma \geq 0$ and the line $\operatorname{Im} \lambda^{-}(\sigma)=0$ crossing the point $\sigma=e^{i \phi_{1}}$. The map is provided by $\lambda^{-}(\sigma)$.

The second sheet and all the next ones are arranged in a very similar way to the corresponding sheets in the even parity energy level case. To achieve the second sheet on which the levels $E_{4}^{-}(\lambda)$ and $E_{5}^{-}(\lambda)$ are defined we should cross a cut $A_{2} / \bar{B}_{2}$ of Fig. 6 in any direction close to the branch point $\lambda_{1}=\lambda^{-}\left(e^{i \phi_{1}}\right)=\phi_{1} / \sin \phi_{1}<0$. The point opens the level $E_{4}^{-}(\lambda)$ on the sheet for $-\propto<\lambda<\lambda_{1}$. There is a second branch point on the sheet at $\lambda=\lambda_{2}=\phi_{2} / \sin \phi_{2}>0$. opening the level $E_{5}^{-}(\lambda)$ for $\lambda_{2}<\lambda<+\propto$. The sheet emerges as a map of an area of Fig. 5 lying between two successive lines $\operatorname{Im} \lambda^{-}(\sigma)=0$, the first one crossing the singular point $\sigma_{1}=e^{i \phi_{1}}$ and the next one crossing the unit circle at $\sigma_{2}=e^{i \phi_{2}}$.

All the next sheets appear as maps of successive areas bounded by corresponding pairs of the lines $\operatorname{Im} \lambda^{-}(\sigma)=0$ crossing the successive singular points $\sigma_{k}=e^{i \phi_{k}}, k=2,3, \ldots$, in the direction of an increasing argument of $\sigma$. However, contrary to the even parity case maps of the negative argument sheets of the $\sigma$-Riemann surface do not produce additional (twin in forms) sheets on the $\lambda$-Riemann surface what corresponds to the absence of relevant right 
cut on the first sheet of the latter surface. It means that the complex conjugated positive and negative argument sheets of the $\sigma$-Riemann surface map into the same sheets of the $\lambda$-Riemann ones for the odd energy function $E^{-}(\lambda)$.

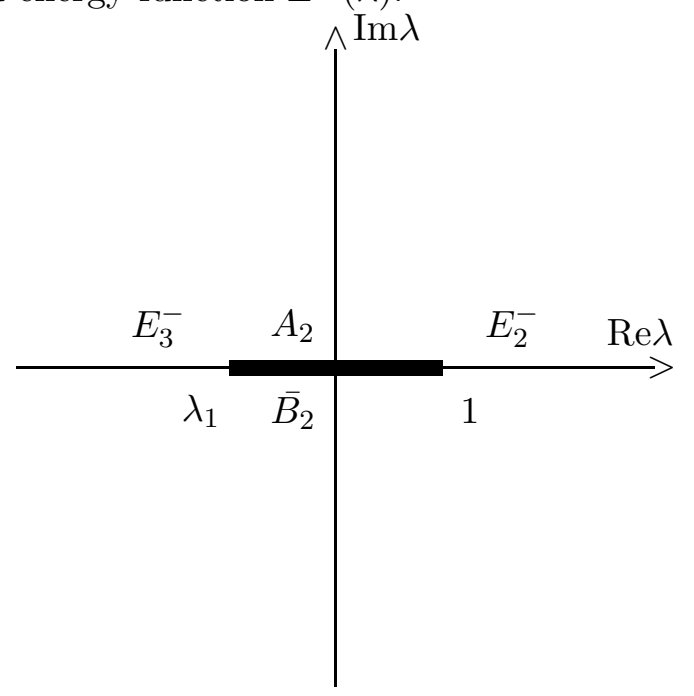

Fig.6 The sheet of the $\lambda$-Riemann surface corresponding to the level $E_{2}^{-}(\lambda)$ and its pseudolevelcompartner $E_{3}^{-}(\lambda)$

\subsection{Relation between analytical properties of energy levels and analytical prop- erties of transmission coefficient}

It is a standard result of the 1-dim quantum mechanics that energy levels of bound states are simple poles for a transmission coefficient of an accompanied 1-dim scattering problem [5]. These poles have to occupy the positions on the positive imaginary axis of the complex momentum corresponding to an infinite motion. In fact this last property is the main criterion for selecting the real bound state energies from the whole set of poles the transmission coefficient can have. Since the analysis of the analytical quantization conditions of the previous section provided us with a variety of the solutions which all have to be poles for a transmission coefficient then to select out of them those being the physical bound state levels we have to discuss roles played by them in the corresponding transmission coefficient.

We will give to our considerations a standard formulation shifting all energy levels by $-\lambda^{2}$ so that the infinite motion takes place for $E>0$ with the momentum $k=\sqrt{E}$ outside the well and with the momentum $k^{\prime}=\left(k^{2}+\lambda^{2}\right)^{1 / 2}$ inside it (the bottom of the well is now at $V=-\lambda^{2}$, of course). Then the reflection (R) and transmission (T) coefficients for the case are the following:

$$
\begin{gathered}
T(k)=\frac{k k^{\prime} e^{-2 i k}}{\left(k \cos k^{\prime}-i k^{\prime} \sin k^{\prime}\right)\left(k^{\prime} \cos k^{\prime}-i k \sin k^{\prime}\right)} \\
R(k)=\frac{i \lambda^{2} \sin 2 k^{\prime}}{2 k k^{\prime}} T(k)
\end{gathered}
$$

It is seen from (2.10) that $\mathrm{T}$ (and $\mathrm{R}$ as well) as a function of complex $k$ is meromorphic with their poles coinciding with roots of the T-denominator. Clearly these poles occupy exactly 
the positions of energy levels in the $k$-plane we have found in the previous paragraphs. The denominator factorization in (2.10) occurs due to the reflection symmetry of the potential well. In particular the first denominator factor in (2.10) corresponds to the odd parity levels and the second to the even ones.

We can separate the investigations of the levels of different parities (according to that we have done earlier) by considering instead of the coefficients (2.10) two pairs of the following ones:

$$
\left\{\begin{array}{l}
T^{+}(k)=\frac{k}{k^{\prime} \cos k^{\prime}-i k \sin k^{\prime}} \\
R^{+}(k)=\frac{i \lambda \cos k^{\prime}}{k} T^{+}(k) \\
T^{-}(k)=\frac{k}{k \cos k^{\prime}-i k^{\prime} \sin k^{\prime}} \\
R^{-}(k)=\frac{i \lambda \sin k^{\prime}}{k} T^{-}(k)
\end{array}\right.
$$

Of course, the two above pairs of coefficients correspond now to two different potential wells: $V^{+}(x, \lambda)$ and $V^{-}(x, \lambda)$ respectively which can be reconstructed with the help of the inverse scattering method [6].

Let us consider first a pole structure of the $k$-plane corresponding to the even parity case. The corresponding analysis of the odd one is analogous.

This structure is shown in Fig. 7 and follows from an observation that $\phi_{l}^{+}(\lambda)$ as a $l^{\text {th }}$ analytical solution to the first of the conditions (2.4) defines a pole of $T^{+}(k)$ on the $k$-plane at $k_{l}^{+}(\lambda)=i \lambda \sin \phi_{l}^{+}(\lambda)$.

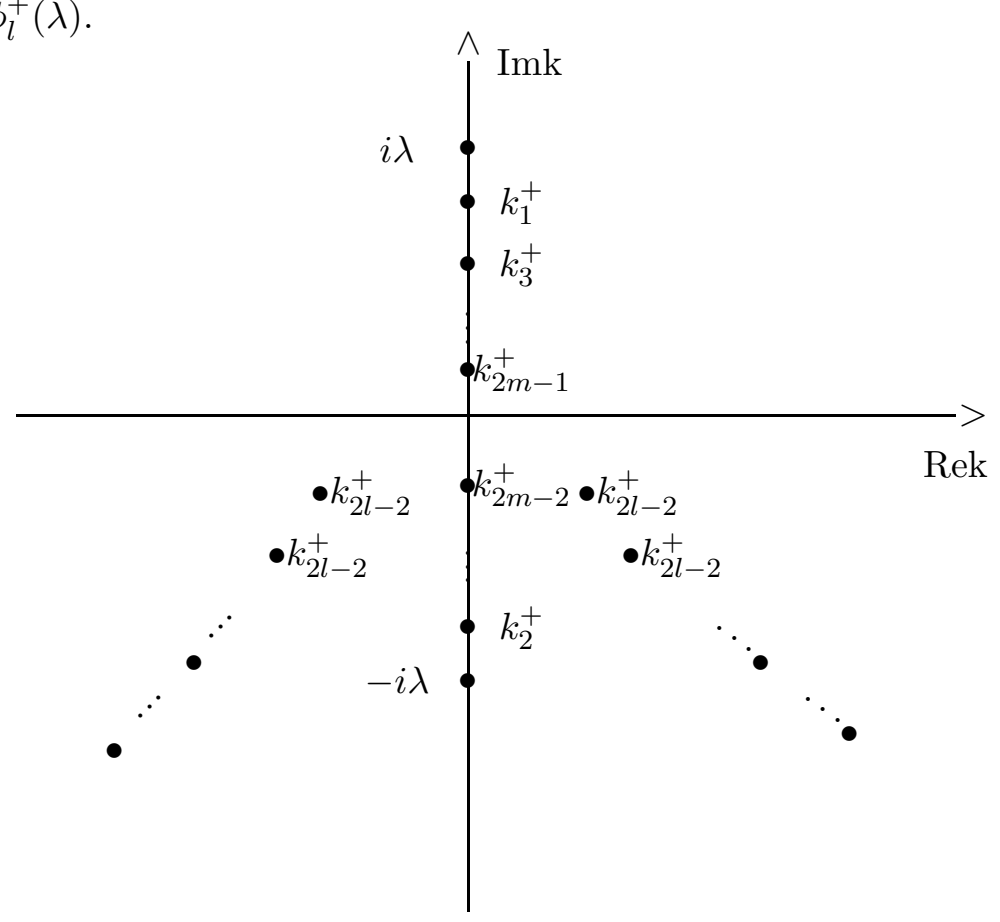

Fig.7 The k-plane of the transmission coefficient $T^{+}(k)$ 
According to our earlier observation together with solutions we have for a given $\lambda>0$ we have to consider also the ones we have for $-\lambda$.

There is a finite number $m\left(\left|\phi_{m}^{+}( \pm \lambda)\right| \leq m \pi \leq \lambda\right)$ of solutions to (2.4) for which $\phi_{l}^{+}( \pm \lambda)$ are real and $\pm \lambda \sin \phi_{m}^{+}( \pm \lambda)>0$. Poles corresponding to these solutions (which include the one for the ground state (for $l=1)$ ) are distributed on the segment $(0, i \lambda)$ of the positive imaginary axis and represent the physical bound states of the potential $V^{+}(x, \lambda)$.

There are also $m-1$ real solutions to (2.4) but with $\pm \lambda \sin \phi_{m}^{+}( \pm \lambda)<0$ generating in that way poles lying in the $k$-plane on the segment $(0,-i)$ (i.e. below the real axis of the plane). Therefore the latter which we call pseudoenergy levels cannot represent the bound states in $V^{+}(x, \lambda)$. These poles appear as branch partners of the previous ones (except the ground state partner which does not exist) which therefore can coincide with the latter at the corresponding pseudothresholds $\lambda=\lambda_{l}$ (see figures 3-4). However, loci of these pseudothresholds in the $k$ plane are just below the real axis not contradicting therefore the physical level nondegeneracy theorem. According to our previous notation (see Fig. $3-4$ ) these $2 m-1$ poles are: $k_{1}^{+}(\lambda)$, $\left(k_{2}^{+}(\lambda), k_{3}^{+}(\lambda)\right),\left(k_{4}^{+}(\lambda), k_{5}^{+}(\lambda)\right), \ldots,\left(k_{2 m-2}^{+}(\lambda), k_{2 m-1}^{+}(\lambda)\right)$ where the branch partners are paired and the poles with odd indices represent the physical levels (lie above the real axis).

Finally, there are infinitely many poles of $T^{+}(k, \lambda)$ lying below the real axis of the $k$ plane on both the sides of the imaginary one and symmetrically with respect to it (due to the relation $k_{l}(\lambda)=k_{l}^{*}\left(\lambda^{*}\right)$ considered for real $\left.\lambda\right)$ but outside of it with finite distances between any two of them and because of this distributed up to infinity. These are of course the poles: $\left(k_{2 l-2}^{+}(\lambda), k_{2 l-1}^{+}(\lambda)\right), l=m+1, m+2, \ldots$, .

If $\lambda$ varies through real positive values increasing all the poles lying outside the segment $(-i \lambda, i \lambda)$ move towards it and a symmetric pair of them $\left(k_{2 l-2}^{+}(\lambda), k_{2 l-1}^{+}(\lambda)\right)$ achieves the segment just for $\lambda$ approaching $\lambda_{l}$ a value being a corresponding pseudothreshold for the pair. While achieving the segment the pair disjoints again with its member $k_{2 l-1}^{+}(\lambda)$ moving upwards imaginary axis and with the member $k_{2 l-2}^{+}(\lambda)$ moving downwards. The first one crosses eventually the real axis becoming a bound state whilst the second one does it never becoming a pseudoenergy level.

The above description works for every pair of the pole partners except the one corresponding to the ground state energy which is single. This for real positive $\lambda$ (as well as for the negative one due to a relation $k_{l}(\lambda)=k_{l}(-\lambda)$ ) valid for any $\lambda$ ) is above the real $k$-axis and thus represents the ground state energy. However, for imaginary $\lambda(\sigma)$, when $0<\sigma<1$ or $1<\sigma<+\propto, E_{1}^{+}(\lambda)=\lambda^{2}$ is real (and of course negative) but $k_{1}^{+}(\lambda)$ has negative imaginary part and therefore $E_{1}^{+}(\lambda)$ cannot represent a bound state. This fits well our intuition since for imaginary $\lambda$ the rectangular well become rather a rectangular barrier excluding of course any bound state.

A similar analysis of the odd parity levels corresponding to the potential $V^{-}(x, \lambda)$ leads us to the following conclusions.

For a given $\lambda>0$ and $m \pi \leq \lambda$ poles: $k_{2}^{-}(\lambda),\left(k_{3}^{-}(-\lambda), k_{4}^{-}(-\lambda)\right),\left(k_{5}^{-}(\lambda), k_{6}^{-}(\lambda)\right),\left(k_{7}^{-}(-\lambda)\right.$, $\left.k_{8}^{-}(-\lambda)\right), \ldots,\left(k_{2 m-1}^{-}\left((-1)^{m-1} \lambda\right), k_{2 m}^{-}\left((-1)^{m-1} \lambda\right)\right)$ lie in the segment $(-i \lambda, i \lambda)$ whilst the remaining ones $\left(k_{2 l-1}^{-}\left((-1)^{l-1} \lambda\right), k_{2 l}^{-}\left((-1)^{l-1} \lambda\right)\right), l=m+1, m+2, \ldots$, lie outside the segment, below the real axis and symmetrically with respect to imaginary axis.

A behaviour of all the paired poles with varying along the positive real axis is exactly the same as in the even parity level case. Only the pole $k_{2}^{-}(\lambda)$ seems to differ with this respect in comparison with $k_{1}^{-}(\lambda)$ escaping to infinity along the negative imaginary axis when $\lambda \rightarrow 0_{+}$. However if one considers a behaviour of the levels rather as functions of $\sigma$ for $\sigma \rightarrow 0_{+}$(or for $\sigma \rightarrow+\propto$ ) on the corresponding first sheets of figures 5 and 2 respectively (this corresponds 
to $\lambda \rightarrow 0_{+}$for the odd level but describes a little bit more complex path for the even one ending however at $\lambda=0$ ) then one can find that both the levels behave identically.

\subsection{Perturbation series for energy levels and their summability}

Since the finite rectangular well considered is a perturbation for the infinite rectangular one when $\lambda^{-2} \rightarrow 0$ then the corresponding energy levels of the former should approach the corresponding levels of the latter potential in this limit. It is interesting that with the previous considerations we are able to conclude that all the corresponding perturbation series expansions are convergent. The corresponding conclusions are in fact obvious for all the energy levels higher than the ground state one. To show this let us consider a pair of levels $E_{4 n+1}^{+}, E_{4 n+2}^{+}$lying on the $2 n+1^{t h}$ sheet shown in Fig. 4 with the level $E_{4 n+1}^{+}$belonging to the even spectrum of the finite rectangular well. A function $E^{+}(\lambda)=\lambda^{2} z^{2}(\lambda)$ being a holomorphic extension of both the levels considered is holomorphic on the $2 n+1^{\text {th }}$ sheet outside any closed contour $\mathrm{C}$ containing the branch points $\lambda=0, \lambda_{2 n+1}$ and $\lambda_{2 n+2}$ inside (see Fig. 4). For $\lambda \rightarrow \propto$ on the sheet $E^{+}(\lambda) \sim E_{4 n+1}^{+}(\lambda) \sim \pi^{2}(8 n+1)^{2} / 4$ and therefore according to the Cauchy theorem we get for $E^{+}(\lambda)$ :

$$
E^{+}(\lambda)=\frac{((8 n+1) \pi)^{2}}{4}-\frac{1}{2 \pi i} \oint_{C} \frac{E^{+}\left(\lambda^{\prime}\right)}{\lambda^{\prime}-\lambda} d \lambda^{\prime}
$$

It follows from $(2.12)$ that the series

$$
\frac{((8 n+1) \pi)^{2}}{4}+\sum_{k \geq 0} \frac{a_{2 n+1, k}}{\lambda^{k+1}}
$$

with

$$
a_{2 n+1, k}=\frac{(-1)^{k}}{2 \pi i} \oint_{C} E^{+}(\lambda) \lambda^{k} d \lambda
$$

which is the perturbative one for the $E_{4 n+1}^{+}(\lambda)$ level is convergent to the level for $|\lambda|>\left|\lambda_{2 n+2}\right|$ i.e. for all $\lambda$ 's just above its pseudothreshold.

For the ground state energy level $E_{1}^{+}(\lambda)$ an analogous conclusion can be obtained by considering instead the cut pattern shown in Fig. 3a the one when the upper and the lower left cut boundaries in the figure are rotated by $\pm \pi$ respectively providing us with a sheet arranged according to Fig. 8. It follows from the figure that the perturbative series for $E_{1}^{+}(\lambda)$ is obtained from (2.12) by putting there $n=1$ and $C=C^{\prime}$ with its convergence radius given by $\max \left(\left|\lambda_{1}\right|,\left|\lambda_{2}\right|\right)$.

The same conclusions can be drawn for the perturbative series constructed for the odd parity energy levels applying exactly the same technique of considerations as we did in the case of the even parity levels. The series constructed for a level $E_{n}^{-}(\lambda)$ is therefore convergent for $|\lambda|$ sufficiently large on the sheet on which the level is defined. 


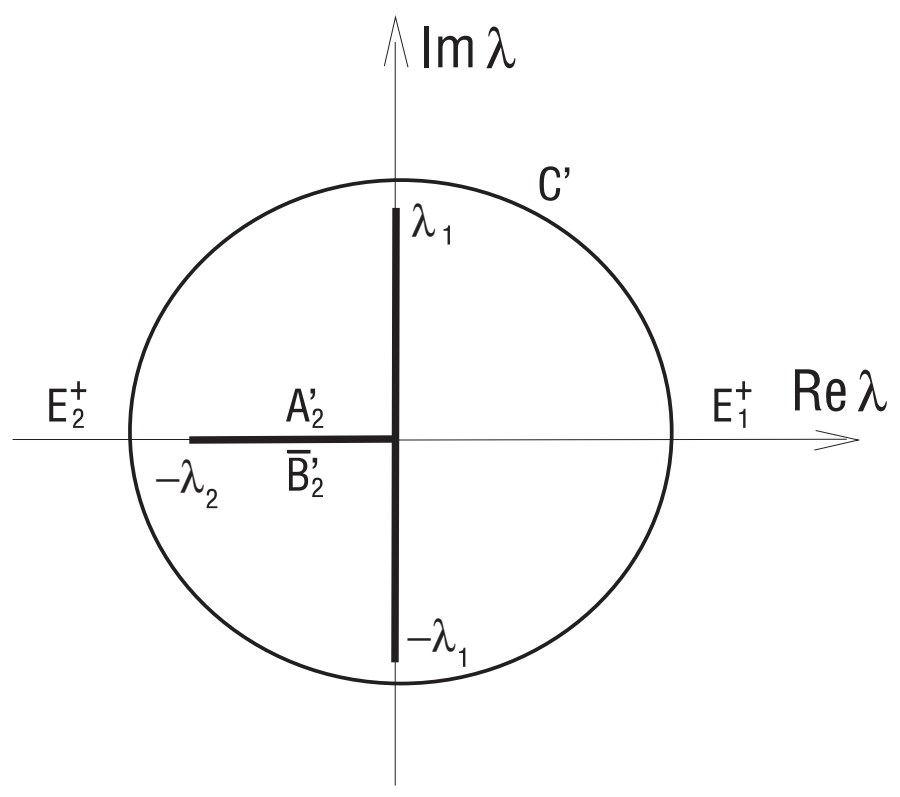

Fig.8 The cut structure of the sheet on which the perturbative series for $E_{1}^{+}(\lambda)$ is studied

\section{Dirac delta barrier as perturbation}

A second example of a perturbation which can be analyzed analytically is provided by the Dirac delta barrier introduced into the infinite rectangular well i.e. it is given by

$$
\left\{\begin{array}{l}
V(x, g)=2 g \delta(x) \quad|x|<1 \\
\psi( \pm 1)=0
\end{array}\right.
$$

where $\psi(x)$ is a wave function for the case. A role of a perturbation parameter is played by g. For $g=0$ we get a problem of the energy spectrum in the infinite rectangular well i.e. an asymptotic limit of any energy level $E_{n}(g)$ of the potential (2.14) is just a corresponding level $E_{n}$ of the rectangular well. In the limit $g \rightarrow+\propto$ we get instead two infinite rectangular wells with the half of that size of the well we started with for which their energy spectra should coincide with the corresponding limit of $E_{n}(g)$. This is not unexpected. What is interesting in this example is the fact [4] that every even parity level of the potential (2.14) is a branch of some ramified function $E(g)$ considered as a function of complex $g$ (odd parity energy levels decouple of $g$ and therefore coincide with the odd ones of the infinite rectangular well). With respect to this property the considered case reminds to some extent the previous one. However there is one considerable difference between them. This is that the existence of all the even energy levels $E_{n}(g)$ does not depend on $g$ i.e. each level exists for any $g$, including $g=0$. Therefore, we cannot expect on a $g$-Riemann surface $\mathbf{R}_{g}$ of $E(g)$ the real branch points to appear having meanings of pseudothresholds for the levels $E_{n}(g)$. On the other hand there are branch points on $\mathbf{R}_{g}$ (as they have to be because $E(g)$ is a ramified function) which are complex but which physical meaning seems to be puzzled. Nevertheless there is also a property of the considered case which is a copy of the corresponding one for the finite rectangular well. Namely, perturbative series for even $E_{n}(g)$ are all convergent. All these 
follow from the quantization condition for even energy levels

$$
\left\{\begin{array}{l}
k \cot k=-g \\
k=\sqrt{E}
\end{array}\right.
$$

The condition (3.1) defines $g$ as a meromorphic function of $k$ with simple poles on the $k$-plane at $k=r \pi, r= \pm 1, \pm 2, \ldots$. Therefore an inversion of the relation (3.1) will be easy if we know positions of zeros of $g^{\prime}(k)$ in the $k$-plane. These positions are determined by (3.1) and by the following condition:

$$
g^{2}+g+k^{2}=0
$$

Both the last equations are equivalent to

$$
\left\{\begin{array}{l}
\sin 2 k=2 k \\
g=-k \cot k
\end{array}\right.
$$

The first of equations (3.3) has a solution for $k=0$ and an infinite number of complex solutions in the $k$-plane (i.e. $\operatorname{Im} k \neq 0$ for all these solutions) with the property that if $k_{l}$, $l=1,2, \ldots$, is a solution to it then $-k_{l}, k_{l}^{*}$ and $-k_{l}^{*}$ are also. It is easy to show that for large $l$ $\operatorname{Im} k_{l}$ increases as $\ln l$ and $\operatorname{Re} k_{l}$ as $l \pi$ [⿴囗十 . A corresponding pattern of lines $\operatorname{Re} g=$ const taking into account the distribution of singular points described above is shown in Fig. 9. The pattern allows us for an easy reproducing of a $g$-Riemann surface $\mathbf{R}_{g}$ for an inverse function $k(g)$. Namely, we can arrange cuts on $\mathbf{R}_{g}$ in such a way to map a $l^{\text {th }}$ dashed area of Fig. 9 into a sheet of $\mathbf{R}_{g}$ corresponding to $E_{l}^{t h}$ even energy level of the potential considered. This is shown in Fig. 10.

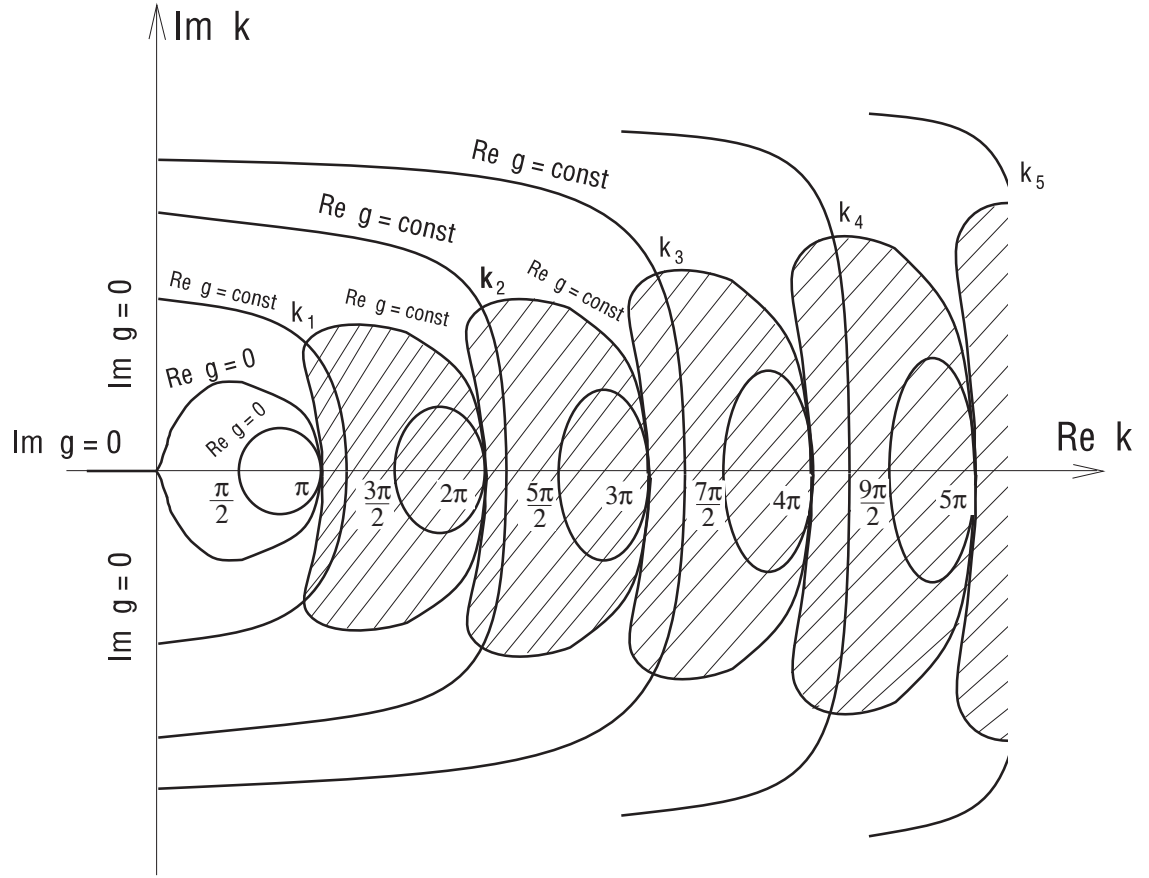

Fig.9 The pattern of lines $\operatorname{Reg}(k)=$ const and $\operatorname{Img}(g)=$ const for $g(k)=-k \cot k$ 
Because $g(k)$ is a symmetric function of $k$ i.e. $g(k)=g(-k)$ with the singular point at $k=0$ (i.e. $g^{\prime}(0)=0$ ) its inverse function $k(g)$ is defined on the Riemann surface $\mathbf{R}_{g}$ which consists of two twin systems of sheets joined by one cut only beginning at $g=-1$ and running to $-\propto$. These two systems of sheets are maps of the right and the left $k$-halfplanes of Fig.9, respectively. The two sheets opening the systems and joined by the cut described above are the ones on which the ground state energy level is defined taking the same values in the corresponding points of the sheets. This is because $E(g)=k^{2}(g)$. Therefore, for $E(g)$ the point $g=-1$ on the first sheet considered is not a branch one and we can consider only one of the two systems of sheets corresponding for example to Rek $\geq 0$ what is assumed from now on.

In accordance with the results of Ushveridze [4] all singular points of $E(g)$ lie on the first sheet corresponding to the ground state energy level of the potential (3.1). The sheet is an image of an area which completes the dashed ones of Fig. 9 to the full right $k$-halfplane and is sketched in Fig. 10.

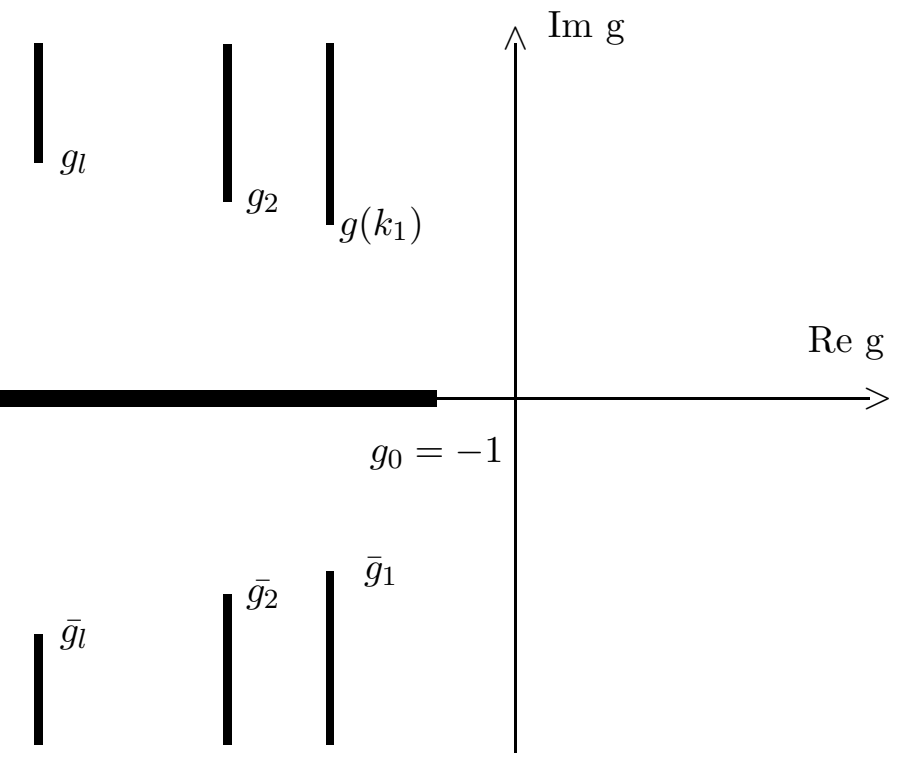

Fig.10 The cut structure of the g-Riemann surface for $\mathrm{k}(\mathrm{g})$ determining the surface

As it has been already explained earlier on the $l^{\text {th }}$ sheet where the $l^{\text {th }}$ energy level $E_{l}(g)$ is defined on there is a unique pair of complex conjugated branch points at $g_{l}\left(=g\left(k_{l}\right)\right)$ and $\bar{g}_{l}$ by which the level contacts with the ground state one (see Fig. 11). The level is holomorphic on its sheet at $g=0$ and approaches $[(l+1) \pi]^{2}$ or $(l \pi)^{2}$ for $g \rightarrow \propto$ in the halfplanes Re $g>$ $\operatorname{Re} g_{l}$ or $\operatorname{Re} g<\operatorname{Re} g_{l}$ respectively.

Because of the holomorphicity of $E_{l}(g)$ at $g=0$ its perturbative series with respect to $g$ converges inside the circle $|g|=\left|g_{l}\right|$.

For large $g$ however we have to consider together with $E_{l}(g)$ also its neighbour $E_{l+1}(g)$ for which its limit for $g$ in the halfplane $\operatorname{Re} g<\operatorname{Re} g_{l}$ is the same as for $E_{l}(g)$ in the halfplane $\operatorname{Re} g>\operatorname{Re} g_{l}$. A common sheet for both the levels is shown in Fig. 12. It is obvious that 
we can apply here the Cauchy integral technique to show the convergence of the asymptotic series for $E_{l}(g)$ and for $E_{l+1}(g)$ for large g with $|g|>\left|g_{l+1}\right|, l=1,2, \ldots$, .

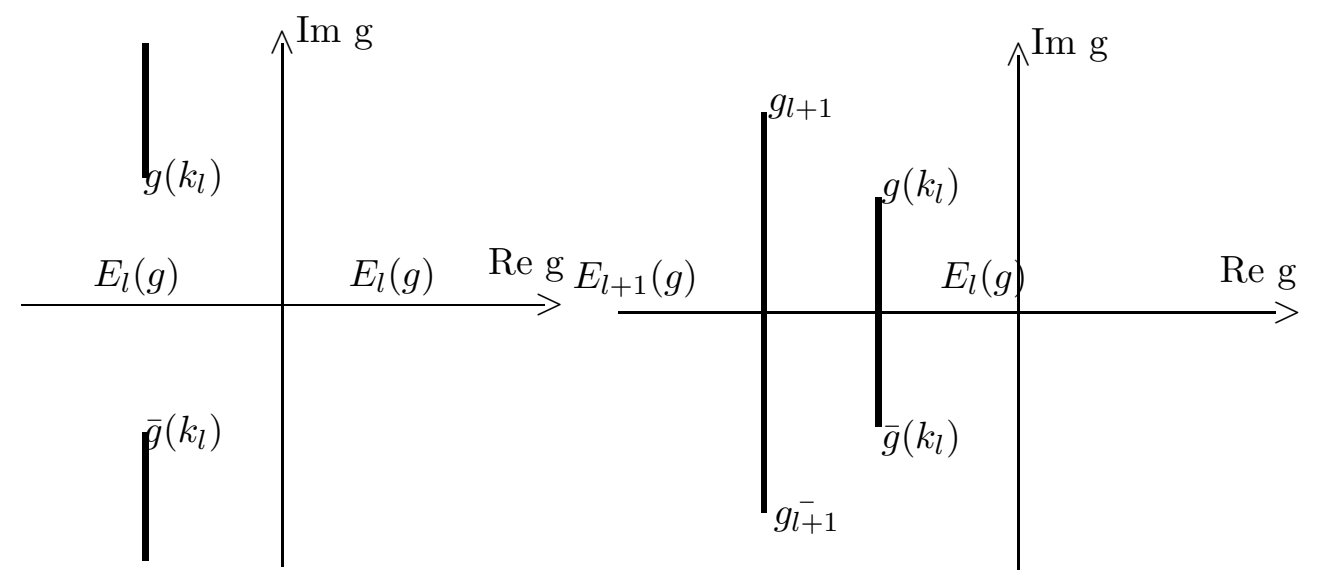

Fig.11 The $g$-Riemann surface for $l^{t h}$ energy Fig.12 The cut structure of the sheet on level of the infinite rectangular well with which the perturbative series for $E_{l}(g)$ is the Dirac delta barrier studied

The corresponding statement for the ground state energy level perturbation series is also true for $|g|>\left|g_{1}\right|$.

\section{Conclusions}

The model of finite rectangular well which we have considered provided us with several properties differing it from the previous ones. First it was a number of energy levels varying with a perturbation parameter thus changing their quality of being real to becoming only potential ones.

Secondly it was the existence of the pseudoenergy levels in the model the main role of which was just to allow the level crossing phenomenon to appear.

It was also an easiness of predictions of the pseudothreshold distribution as a part of the level crossing loci. The latter property followed however from the simplicity of the quantization conditions for the case as illustrated by Fig. 1. In fact the remaining three branch points, the two for the ground state level at $\lambda= \pm i$ and the logarithmic one for all the levels at $\lambda=0$ could be identified only by detailed calculations.

Finally it was the convergence of perturbation series for large $\lambda$ and for all the levels. This property could not be deduced prior to the detailed knowledge of the $\lambda$-Riemann surface topology just because for imaginary $\lambda$ (no matter small or large) the rectangular well became the rectangular barrier suggesting rather a possibility for the perturbation series to be divergent in these directions because of a repelling character of the barrier. However the repulsion of the rectangular barrier appears to be not enough strong to destroy the convergence of the perturbation series. 
The property of the perturbation series to be convergent was shared also by the corresponding series constructed for the levels in the infinite rectangular well perturbed by the Dirac delta barrier.

Generally the analytical properties of energy levels considered above show that they depend strongly on the model used. Crossing of levels considered as the main cause for the presence of branch points in the dependence of levels on a chosen perturbation parameter seems to be as obvious as puzzled in most of the models. In particular these are the positions of branch points in investigated models which does not seem to be covered by some universal rules. This latter statement is true also in the case of the rectangular well energy levels investigated in this paper and the name of pseudothresholds we gave to the underlying branch points cannot be considered more seriously than only a convenient convention. It seems rather that such a large variety of different parameters which were used as perturbation ones in models considered so far does not allow us to formulate some common rules covering the distributions of branch points and giving them in this way some common sense. Only the most general hints such as for example the non existing of energy level degeneracy in the 1-dim quantum mechanics can help us to make some predictions. In most cases however only a detailed knowledge of considered potentials and corresponding quantization conditions the former generate can allow us to formulate some reasonable predictions prior to detailed calculations.

\section{References}

[1] Bender C.M. and Wu T.T., Phys. Rev. 184 (1969) 1231, Phys.Rev. D7 (1973) 1620

[2] Giller S., Acta Phys. Pol. B23 (1992) 457-511

[3] Simon B. and Dicke A., Ann. Phys. (N.Y.) 58 (1970) 76

[4] Ushveridze A. G.,J. Phys. A: Math. Gen. 21 (1988) 955-970

[5] Landau L.D. and Lifshitz E.M., Quantum Mechanics. Nonrelativistic Theory (Oxford, New York: Pergamon Press 1965)

[6] Zacharov V.E., Manakov S.V., Novikov S.P., Pitayevski L.P., Soliton Theory (Moscow: Nauka 1980) (in Russian)

[7] Ushveridze A.G.,Quasi-Exactly Solvable Problems in Quantum Mechanics, IOP Publishing (1994)

[8] Banks T.I. and Bender C.M., J.Math. Phys. 13 (1972) 1320

[9] Bender C.M., Happ H.J. and Svetitsky B., Phys. Rev. D8 (1974) 2324

[10] Shanley P.E. Phys.Lett. A117 (1986) 161 